REVIEW ARTICLE

\title{
A Consideration of Antibacterial Agent Efficacies in the Treatment and Prevention of Formation of Staphylococcus aureus Biofilm
}

\author{
Zahra Sedarat $^{1}$, Andrew W. Taylor-Robinson ${ }^{2}$ \\ ${ }^{1}$ Cellular \& Molecular Research Center, Shahrekord University of Medical Sciences, Shahrekord, Iran \\ ${ }^{2}$ Infectious Diseases Research Group, School of Health, Medical \& Applied Sciences, Central Queensland University, \\ Brisbane, Australia
}

\begin{abstract}
Staphylococcus aureus is a Gram-positive bacterium found frequently on a person's skin and sometimes in their upper respiratory tract. Although regarded primarily as a commensal of the human microbiota $S$. aureus shows the ability to become an opportunistic pathogen. Hence, it is a common cause of skin and lung infections and of food poisoning. S. aureus forms biofilms, complex communities of bacteria inside an exopolysaccharide matrix, which adhere to different surfaces, including those associated with hospital-acquired infections such as catheters, shunts and other implanted medical devices. In this instance, the presence of proteins adsorbed to the surface of the biomaterial provides a nutrient source for bacterial growth.

Due to antimicrobial resistance, use of longstanding antibiotics alone is increasingly an ineffective therapeutic intervention for biofilm-related infections. Therefore, a growing concern is the treatment of medical devices in order to prevent antibiotic resistance associated with routine handling of these items in a healthcare setting. Consequently, several different biotechnological approaches have targeted a practical solution to $S$. aureus biofilm formation. These include novel antibiotics administered alone or combined with other compounds, application of natural products like enzymes and antimicrobial peptides, and harnessing of nanoparticles and phage therapy. This brief article provides an overview of each of these cutting-edge methods aimed at inhibition of $S$. aureus biofilms. Development of an effective agent to prevent and treat biofilm formation would represent a significant step forward for infection control of methicillin-resistant $S$. aureus (MRSA) and other antibiotic-resistant strains that provides a major global public health challenge. J Microbiol Infect Dis 2019; 9(4):167-172.
\end{abstract}

Keywords: Staphylococcus aureus, bacterium, biofilm, infection, antibiotic, resistance

\section{INTRODUCTION}

Biofilm formation is an important contributory factor in the establishment and persistence of bacterial infections, and thus is considered a principal reason for antimicrobial resistance [1]. Fossils belonging to different domains of microorganism, archaea and bacteria, and which date back over three billion years have been shown to contain biofilm. Evidently, biofilm formation is an archaic component of the prokaryotic life cycle as well as a critical determinant of survival across a broad spectrum of niche environments [2]. A biofilm is composed of a self-produced extracellular matrix (ECM) made of exopolysaccharide, commonly described as 'slime', that encloses a community, or colony, of microorganisms. The slime consists of multiple porous layers that contain channels to enable cells in the centre of the colony to receive nutrients and to remove metabolic waste products. Within this elaborate structure antimicrobial resistance is greatly enhanced [3]. Survival in hostile environments, including being subjected to host innate and adaptive immunity, is improved, thereby leading to chronic infection and inflammation. The production of bacterial biofilms is thought to be responsible for over $80 \%$ of persistent clinical infections, for which conventional treatments are no longer effective at achieving clearance [4].

Correspondence: Prof. Andrew W. Taylor-Robinson, School of Health, Medical \& Applied Sciences, Central Queensland University, 160 Ann Street, Brisbane, QLD 4000, Australia. Tel: +61 73295 1185; 


\section{BIOFILM FORMATION}

Catheters, shunts and other implanted medical devices provide suitable surfaces on which biofilms formed by both Gram-negative and Gram-positive bacteria can develop [5,6]. Most of these bacteria form part of a healthy person's microbiome, existing as commensals or in a mutually beneficial relationship with their host usually in the saliva, mouth, gastrointestinal tract, nose, ear canal, mucosa or on the skin where they help in numerous metabolic activities. However, they retain the potential for pathogenesis and, given the opportunity, their unregulated growth or presence in an atypical location can lead to infection [7]. The species most frequently detected are Staphylococcus aureus, S. epidermidis, Streptococcus viridans, Escherichia coli, Enterococcus faecalis, Klebsiella pneumoniae, Proteus mirabilis and Pseudomonas aeruginosa [6]. The first two of these, the Gram-positive cocci S. aureus and S. epidermidis, together are estimated to cause between $50-70 \%$ of catheter biofilm infections, around $40-50 \%$ of prosthetic heart valve infections and over $85 \%$ of septicemia cases [5].

In the process of being used for their intended purpose in a healthcare setting medical devices and biomaterials are prone to contacting - and becoming covered by deposits of - naturallyoccurring proteins on or in a patient's skin, tissues or body fluids [6]. This applies especially to blood plasma and serum proteins such as fibronectin, fibrinogen, thrombospondin and vitronectin, all of which may provide a platform for ECM formation [8]. The adherence by ECM components to serum proteins is a fundamental initial stage in the interaction between $S$. aureus and a human host. Once established on the underlying substrate, this bacterium employs a wide range of modes to facilitate colonization and dissemination [9].

There are five main steps involved in biofilm formation; in sequence these are attachment, multiplication, exodus, maturation and dispersal. Following alighting upon a surface, bacteria produce various cell proteins to enable steadfast adherence to different host matrix substrates. The next step, multiplication, happens in the nutrient-rich conditions provided by the presence of serum proteins, when the complex of $S$. aureus cells begins to divide and accumulate.
Approximately six hours after the initiation of multiplication, these cells start to be released, a process called exodus, which is followed by maturation. At this step, a microcolony structure will form, which further increases contact with the underlying substrate. Finally, when threshold cell density is reached, the level of which varies with conditions, triggers autogenous induction of the production of dispersal cells [10]. This process is under the command of $S$. aureus accessory gene regulator quorum sensing, a cell-to-cell communication system that controls expression of many genes in response to bacterial population density $[7,10]$.

\section{CHALLENGES TO TARGETING BIOFILMS}

Inasmuch as biofilms maintain a barrier to effectively shield bacteria from potentially hostile conditions, their formation provides an extra degree of complexity to the challenge of overcoming antimicrobial resistance [11]. Biofilm bacteria are phenotypically distinct from their isogenic, free-floating planktonic bacteria counterparts by, for instance, actively expressing extracellular proteins, virulence factors and surfactants [12]. Treatments that are developed using only planktonic $S$. aureus (such as antibiotic susceptibility testing, performed routinely in diagnostic laboratories) are thus unlikely to be effective against the biofilm phenotype. Moreover, treating $S$. aureus biofilms based on the minimum inhibitory concentration (MIC) that suppresses growth of planktonic phenotypes may actually promote the emergence of antibiotic-resistant subpopulations within the ECM [13]. Even methicillin (MET)sensitive strains may tolerate exposure to considerably higher concentrations of antibiotic, often exceeding those dosages that are achievable clinically without cytotoxic sideeffects, thereby making these infections difficult to eradicate [14].

It is now acknowledged that biofilms are responsible for most chronic staphylococcal infections, especially those that are associated with indwelling medical devices, a mainstay of modern surgical practice [15]. Since $S$. aureus can successfully colonize and establish biofilms in different host environments (notably, prosthesis and implant surfaces, and tissues such as lung, bone and skin), this is a primary consideration in the design of therapies against 
infection. However, the majority of treatments that are currently approved for clinical administration are applicable only to planktonic or acute S. aureus infections [16]. Hence, there is a pressing, and as yet unfulfilled, need for new therapeutic strategies to target biofilmdwelling $S$. aureus. Novel agents under consideration and progress made in their development to date are discussed below.

\section{TREATMENT FOR STAPHYLOCOCCUS AUREUS BIOFILM}

Clinical case reports of biofilms associated with $S$. aureus infections have increased in number over recent years, a disquieting trend that makes it imperative to find novel therapies. Overuse and misuse of frontline antibiotics to combat $S$. aureus has led to the global emergence of multi-drug resistance, notably to MET, which makes it a difficult-to-treat infection. MET-resistant $S$. aureus (MRSA) can form biofilms, a property that underpins its virulence. This is of considerable relevance to hospitals and other healthcare facilities, settings in which MRSA is particularly prevalent and where it is associated with reduced clinical outcomes [17]. Therefore, a range of efforts has been made to ameliorate this major public health concern [18].

\section{Antibiotics}

Several approaches both to prevent and to treat $S$. aureus biofilm infections have been adopted. Based on recent findings MIC, MBIC (the minimum concentration of a chemical, usually a drug, that prevents visible biofilm formation) and MBEC (the minimum concentration necessary to eradicate preformed biofilm) each vary dependent on the antibiotic tested [18]. Other factors such as the apparent age of the biofilm (young or mature) should also be considered. Although rapid diagnosis of biofilm-producing infections is challenging early detection has a substantial influence on the type and effectiveness of treatment [19]. In general, combination therapy of more than one antibiotic, each from a different class, is far more effective than administration of a single antibiotic [20]. It is also asserted that, dependent on concentrations used, azithromycin (AZM, a broad-based antibiotic) and vancomycin (VAN, an agent of last resort) can both exert not an inhibitory influence but instead an inducing effect on biofilm formation [21].
In antibiotic sensitivity tests using the agar dilution method, clinically isolated strains were totally resistant to erythromycin, $75 \%$ resistant to trimethoprim/sulfamethoxazole, clindamycin and ciprofloxacin (CIP), 50\% resistant to gentamicin (GEN), but completely susceptible to amikacin and tetracycline [22]. In another study various combinations of antibiotics were shown to be bacteriostatic or bactericidal to $S$. aureus strains. AZM along with fusidic acid (FA) and oxacillin (OXA) are effective when administered together against MET-sensitive $S$. aureus (MSSA). Cefazolin (CFZ), FA, GEN, OXA and rifampicin (RIF) is another group that displays antibacterial synergism. Other combinations with a similar bacteriostatic or bactericidal effect towards MSSA are AZM, CFZ, CIP and FA; AZM, FA and VAN; CFZ, GEN, RIF and VAN; AZM, CIP and FA; FA and GEN; and of RIF and VAN. On the other hand, only the combinations of CIP, FA and RIF and of FA, RIF and VAN are bactericidal against MRSA biofilms [23].

\section{Natural products}

Several different human body products are known to be generated as an innate immune defence against $S$. aureus biofilm. RNAlllinhibiting peptide and the human cathelicidin peptide LL-37 and its synthetic mimetic D-LL-37 are examples of anti-microbial peptides (AMPs) that disrupt biofilm through either disturbance of embedded cells or its matrix, interruption of its signalling system or alteration in gene regulation of bacteria [18,24]. Enzymes including proteases, deoxyribonucleases and the metalloendopeptidase lysostaphin exhibit antibiofilm properties such as rapidly disconnecting $S$. aureus biofilm from its substratum and impeding its formation [25]. While each of these enzymes involves a distinct biochemical pathway, when used in harness the effect is synergistic, so the overall antimicrobial activity increases commensurately [24].

In addition, phytochemicals and ethanol extracts from medicinal plants such as Kaempferia rotunda L., Caesalpinia sappan L. and Cinnamomum burmanii Nees ex $\mathrm{Bl}$ show inhibitory properties towards $S$. aureus biofilm growth $[18,26]$. These Indonesian plants provide an unusual, but probably not exclusive, botanical source of putative anti-biofilm agents. 
Although it may seem that of these approaches the use of AMPs is the most promising, for both this and a strategy based on phytochemicals extrapolating from in vitro studies may not be an entirely reliable indicator of the human condition. Therefore, testing in vivo on an experimental model is a prerequisite to undertaking trials in volunteers [27,28], a focus for future research.

Antibodies are produced by the humoral arm of the immune system in response to antigenspecific stimulation. Vaccine design can be predicated on artificially inducing generation of host-protective immunoglobulins. Hence, the development of novel vaccines that stimulate a rapid and enhanced antibody response to $S$. aureus antigens is considered a potentially effective tool to combat antibiotic-resistant infections [29]. Possible target antigens are key components of the bacterial cell structure or metabolism and include capsular polysaccharide, clumping factor-A and $-B$, fibronectin-binding protein and ATP-binding cassette transporter. While several vaccine prototypes have passed preclinical development, to date no phase III clinical trial has been completed successfully [30]. Thus, despite considerable research investment - and significant progress - there remains no commercially available vaccine against $S$. aureus.

\section{Other treatments}

As the extent of the prevalence of $S$. aureus infections and the resistance of biofilms to standard antibiotic regimens have become increasingly apparent, endeavors to attain a solution have expanded. The nano-packaging of materials, such as inert metals, that have demonstrable antimicrobial effects is viewed as a practical means to treat biofilms in parallel to the use of antibiotics [31]. Nanoparticles of various compositions have been synthesized and tested against $S$. aureus biofilm with qualified success. In one study, $\mathrm{Ag}_{3} \mathrm{PW}_{12} \mathrm{O}_{40}$ nanoparticles were shown to cause damage to the peptidoglycan component of the Grampositive cell membrane and to down-regulate biofilm-related gene expression [32].

The effectiveness towards $S$. aureus of an antibiotic, either in combination or monotherapy, is reported to improve if administered as a conjugate with nanoparticles made of materials that have antibacterial properties like elemental silver, zinc oxide and titanium dioxide [33,34]. The exciting potential of this mode of co-delivery requires further investigation.

Phage therapy has emerged as an effective means to treat biofilm infections because of its selective specificity. In recent times significant advances have been made in this area [35]. For example, LysCSA13, an endolysin from the $S$. aureus-virulent bacteriophage $\mathrm{CSA}_{13}$, can effectively remove staphylococcal biofilms from various surfaces, raising the possibility that LysCSA13 may be utilised as a promising infection control agent [36]. A potential concern of this strategy is that in some patients it may provoke a host-versus-phage inflammatory immune response. This may result in phage neutralization and thereby impair therapeutic efficacy [37]. Thus, any clinical trial to test the suitability of this phage will be diligent to this possibility.

\section{Conclusion}

Biofilm is a thick extracellular polysaccharide matrix the formation of which is a property of several bacterial human opportunistic pathogens, notably members of the Grampositive genus Staphylococcus. The resilience characteristics of cell adhesion and aggregation that are intrinsic to biofilm promote bacterial survival and growth, while its slime-like coating heightens resistance to antibiotics. Collectively, these factors contribute to persistence of infection. The adherence of biofilms, typically of $S$. aureus, to commonly used medical devices such as catheters constitutes a serious challenge to the control and prevention of hospital-acquired infections. For medically important bacteria, including MRSA, that show high levels of resistance to frontline antimicrobial therapies, it is an increasing imperative to identify and develop effective alternative ways to combat biofilm formation. An example of the public health relevance of this issue is that of an increasingly ageing, and therefore immunologically weakened, population in many industrialized nations worldwide that requires implants and prostheses. When undergoing surgery such patients are rendered particularly vulnerable to $S$. aureus biofilm infection, including MRSA. 
New translational approaches under development include the judicious use of novel antibiotics in various combinations, as well as other agents with anti-biofilm properties such as phytochemicals, AMPs and enzymes. To date, most of these treatments have been tested under in vitro conditions only, and thus their suitability for patient application requires prior assessment in vivo in experimental models and clinical trials. This also applies to the utilisation of nanoparticles and phage therapy. These technologies may achieve optimal efficacy when used in synergy with another remedy, most probably antibiotics. In order to find the most efficacious antibiotic regimen in any given hospital setting it is crucial to evaluate antibiotic resistance and to use this local information to choose a suitable treatment regimen. In this regard, it is a fundamental issue to examine the effect on biofilm formation of antibiotics that are prescribed routinely by physicians for chronic staphylococcal infections such as MRSA.

\section{ACKNOWLEDGMENTS}

Declaration of Conflicting Interests: The authors declare that they have no conflict of interest.

Funding: None declared.

\section{REFERENCES}

1. Hacioglu M, Haciosmanoglu E, Birteksoz-Tan AS, Bozkurt-Guzel C, Savage PB. Effects of ceragenins and conventional antimicrobials on Candida albicans and Staphylococcus aureus mono and multispecies biofilms. Diagn Microbiol Infect Dis 2019: 114863.

2. Hall-Stoodley L, Costerton JW, Stoodley P. Bacterial biofilms: from the natural environment to infectious diseases. Nat Rev Microbiol 2004; 2(2): 95-108.

3. Olsen I. Biofilm-specific antibiotic tolerance and resistance. Eur J Clin Microbiol Infect Dis 2015; 34(5): 877-886.

4. Li X-H, Lee J-H. Antibiofilm agents: A new perspective for antimicrobial strategy. J Microbiol 2017; 55(10): 753-766.

5. Chen $M, Y u Q$, Sun $H$. Novel strategies for the prevention and treatment of biofilm related infections. Int J Mol Sci 2013; 14(9): 18488-18501.

6. Khatoon Z, McTiernan CD, Suuronen EJ, Mah T$F$, Alarcon El. Bacterial biofilm formation on implantable devices and approaches to its treatment and prevention. Heliyon 2018; 4: e01067.

7. Taylor-Robinson A. You scratch my back...the beneficial (and not so beneficial) relationships between organisms. The Conversation. 15 July 2016. Available at: http://theconversation.com/you-scratch-my-backthe-beneficial-and-not-so-beneficial-relationshipsbetween-organisms-57120

8. Lundberg F, Tegenfeldt JO, Montelius L, et al. Protein depositions on one hydrocephalus shunt and on fifteen temporary ventricular catheters. Acta Neurochir 1997; 139(8): 734-742.

9. Clarke SR, Foster SJ. Surface adhesins of Staphylococcus aureus. Adv Microb Physiol 2006; 51: 187-224.

10. Moormeier DE, Bayles KW. Staphylococcus aureus biofilm: a complex developmental organism. Mol Microbiol 2017; 104(3): 365-376.

11. Craft KM, Nguyen JM, Berg LJ, Townsend SD. Methicillin-resistant Staphylococcus aureus (MRSA): antibiotic-resistance and the biofilm phenotype. Med Chem Comm 2019; 10(8): 1231 1241.

12. Secor PR, James GA, Fleckman P, Olerud JE, McInnerney K, Stewart PS. Staphylococcus aureus biofilm and planktonic cultures differentially impact gene expression, mapk phosphorylation, and cytokine production in human keratinocytes. BMC Microbiol 2011; 11(1): 143.

13. Mohammed YHE, Manukumar HM, Rakesh KP, Karthik CS, Mallu P, Qin HL. Vision for medicine: Staphylococcus aureus biofilm war and unlocking key's for anti-biofilm drug development. Microb Pathog 2018; 123: 339-347.

14. Bhattacharya M, Wozniak DJ, Stoodley P, HallStoodley L. Prevention and treatment of Staphylococcus aureus biofilms. Expert Rev Anti Infect Ther 2015; 13(12): 1499-1516.

15. Parvizi J, Pawasarat IM, Azzam KA, Joshi A, Hansen EN, Bozic KJ. Periprosthetic joint infection: the economic impact of methicillinresistant infections. J Arthroplasty 2010; 25(6 Suppl): 103-107.

16. Tong SY, Davis JS, Eichenberger E, Holland TL, Fowler VG Jr. Staphylococcus aureus infections: epidemiology, pathophysiology, clinical manifestations, and management. Clin Microbiol Rev 2015; 28(3): 603-661.

17. Hassoun A, Linden PK, Friedman B. Incidence, prevalence, and management of MRSA bacteremia across patient populations - a review of recent developments in MRSA management and treatment. Crit Care 2017; 21: 211.

18. Suresh MK, Biswas R, Biswas L. An update on recent developments in the prevention and 
treatment of Staphylococcus aureus biofilms. Int $\mathrm{J}$ Med Microbiol 2019; 309(1): 1-12.

19. Wu H, Moser C, Wang H-Z, Høiby N, Song Z-J. Strategies for combating bacterial biofilm infections. Int J Oral Sci 2014; 7(1): 1-7.

20. Herrmann G, Yang L, Wu $\mathrm{H}$, et al. Colistintobramycin combinations are superior to monotherapy concerning the killing of biofilm Pseudomonas aeruginosa. J Infect Dis 2010; 202(10): 1585-1592

21. Majidpour A, Fathizadeh S, Afshar M, et al. Dosedependent effects of common antibiotics used to treat Staphylococcus aureus on biofilm formation. Iran J Pathol 2017; 12(4): 362-370.

22. Rezaei M, Moniri R, Mousavi SGA, Jabari Shiade $M$. Prevalence of biofilm formation among methicillin resistance Staphylococcus aureus isolated from nasal carriers. Jundishapur $J$ Microbiol 2013; 6(6): e9601.

23. Saginur R, Stdenis M, Ferris W, et al. Multiple combination bactericidal testing of staphylococcal biofilms from implant-associated infections. Antimicrob Agents Chemother 2006; 50(1): 55-61.

24. Yasir M, Willcox MDP, Dutta D. Action of antimicrobial peptides against bacterial biofilms. Materials 2018; 11(12): 2468.

25. Craigen B, Dashiff A, Kadouri DE. The use of commercially available alpha-amylase compounds to inhibit and remove Staphylococcus aureus biofilms. Open Microbiol J 2011; 5: 21-31.

26. Pratiwi SUT, Lagendijk EL, Hertiani T, de Weert S, Van Den Hondel CAMJJ. Antimicrobial effects of Indonesian medicinal plant extracts on planktonic and biofilm growth of Pseudomonas aeruginosa and Staphylococcus aureus. J Hortic 2015; 2: 119.

27. Grassi L, Batoni G, Ostyn L, et al. The antimicrobial peptide lin-SB056-1 and its dendrimeric derivative prevent Pseudomonas aeruginosa biofilm formation in physiologically relevant models of chronic infections. Front Microbiol 2019; 10: 198.

28. Raut JS, Karuppayil SM. Phytochemicals as inhibitors of Candida biofilm. Curr Pharm Des 2016; 22(27): 4111-4134.

29. Stentzel S, Gläser R, Bröker BM. Elucidating the anti-Staphylococcus aureus antibody response by immunoproteomics. Proteom Clin Appl 2016; 10(9-10): 1011-1019.

30. Redi D, Raffaelli CS, Rossetti B, De Luca A, Montagnani F. Staphylococcus aureus vaccine preclinical and clinical development: current state of the art. New Microbiol 2018; 41(3): 208-213.

31. Zhou $\mathrm{K}$, Li C, Chen D, et al. A review on nanosystems as an effective approach against infections of Staphylococcus aureus. Int $J$ Nanomed 2018;13: 7333-7347.
32. Liang Z, Qi Y, Guo S, Hao K, Zhao M, Guo N Effect of AgWPA nanoparticles on the inhibition of Staphylococcus aureus growth in biofilms. Food Control 2019; 100: 240-246.

33. Masurkar SA, Chaudhari PR, Shidore VB, Kamble SP. Effect of biologically synthesised silver nanoparticles on Staphylococcus aureus biofilm quenching and prevention of biofilm formation. IET Nanobiotechnol 2012; 6(3): 110-114.

34. Gangadoo S, Taylor-Robinson AW, Chapman J. Nanoparticle and biomaterial characterisation techniques. Mater Technol: Adv Biomater 2015. 30(suppl. 5): B44-B56.

35. Lin DM, Koskella B, Lin HC. Phage therapy: An alternative to antibiotics in the age of multi-drug resistance. World J Gastrointest Pharmacol Ther 2017; 8(3): 162-173.

36. Cha Y, Son B, Ryu S. Effective removal of staphylococcal biofilms on various food contact surfaces by Staphylococcus aureus phage endolysin LysCSA13. Food Microbiol 2019; 84: 103245.

37. Freyberger HR, He Y, Roth AL, Nikolich MP, Filippov AA. Effects of Staphylococcus aureus bacteriophage $\mathrm{K}$ on expression of cytokines and activation markers by human dendritic cells in vitro. Viruses 2018; 10(11): 617. 